\title{
Development of cluster-jet targets: From COSY-11 to FAIR.
}

\author{
A. Täschner, S. General, J. Otte, T. Rausmann and A. Khoukaz \\ Institut für Kernphysik, Wilhelm-Klemm-Str. 9, D-48149 Münster, Germany
}

\begin{abstract}
The development of cluster-jet targets of Münster type is presented. Starting with the first target installed at the COSY-11 experiment the progress is described which was made at a cluster-jet target facility installed in Münster leading to a prototype for a cluster-jet target for the upcoming PANDA experiment at FAIR.
\end{abstract}

Keywords: Cluster target, Internal target, Hydrogen clusters, Nozzle, Laval nozzle, Cryopump PACS: 29.25.Pj,61.46.Bc,61.46.Df

\section{THE CLUSTER-JET TARGET FOR COSY-11}

The COSY-11 experiment [1] is an internal storage ring experiment at the cooler synchrotron COSY at the FZ Jülich. Its main purpose was the investigation of the meson production close to the production threshold in proton-proton and proton-deuteron collisions.

Several design goals had to be achieved during the development of the target for COSY-11. At first the target had to provide a small and precisely known interaction region with the accelerator beam in order to allow for a precise momentum reconstruction of the ejectiles. This implied directly that the influence of the vacuum in the storage ring had to be small and confined to the target region. The target thickness had to be small enough in order to achieve beam lifetimes in the order of $10 \mathrm{~min}$ to $1 \mathrm{~h}$ but on the other hand it had to be large enough in order to provide acceptably high event rates. These constraints ruled out the use of gas-jet targets or solid targets leading to the decision to build a cluster-jet target. In such targets hydrogen or deuterium gas is cooled down to temperatures of $\sim 30 \mathrm{~K}$ and is pressed through a thin laval nozzle. The gas expands into a vaccuum chamber where a small fraction of the gas condensates to nanoparticles called clusters. Using several skimmers a cluster-jet is formed which traverses the accelerator beam with a small angular divergence given by the skimmer geometry.

Since the target facility had to be installed into a gap of $30 \mathrm{~cm}$ between a dipole and a quadrupole magnet of the storage ring, it was not possible to use cluster-jet designs which were in operation at other storage rings at that time. The cluster-jet target of the Münster type [2] uses an innovative pumping scheme with specially designed cryopumps in order to meet these space requirements. After the construction and successful tests in Münster it was transferred to Jülich where it was reassembled. In 1995 it was installed at the COSY-11 place where it worked very reliable and met all demands up to the end of the experiment in 2006. 
TABLE 1. Typical operation parameters of hydrogen cluster-jet targets.

\begin{tabular}{|c|c|c|c|c|}
\hline & CELSIUS & $\begin{array}{c}\text { E835 } \\
\text { FERMILAB }\end{array}$ & $\begin{array}{c}\text { ANKE } \\
\text { COSY-11 }\end{array}$ & Münster \\
\hline nozzle diameter & $100 \mu \mathrm{m}$ & $37 \mu \mathrm{m}$ & $11-16 u m$ & $16-28 \mu \mathrm{m}$ \\
\hline gas temperature & $20-35 \mathrm{~K}$ & $20-32 \mathrm{~K}$ & $22-35 \mathrm{~K}$ & $20-35 \mathrm{~K}$ \\
\hline gas pressure & $1.4 \mathrm{bar}$ & $<10$ bar & 18 bar & $>18 \mathrm{bar}$ \\
\hline distance from nozzle & $0.32 \mathrm{~m}$ & $0.26 \mathrm{~m}$ & $0.65 \mathrm{~m}$ & $2.1 \mathrm{~m}$ \\
\hline target thickness & $1.3 \cdot 10^{14} \mathrm{~cm}^{-2}$ & $3 \cdot 10^{14} \mathrm{~cm}^{-2}$ & $>10^{14} \mathrm{~cm}^{-2}$ & $>4 \cdot 10^{14} \mathrm{~cm}^{-2}$ \\
\hline
\end{tabular}

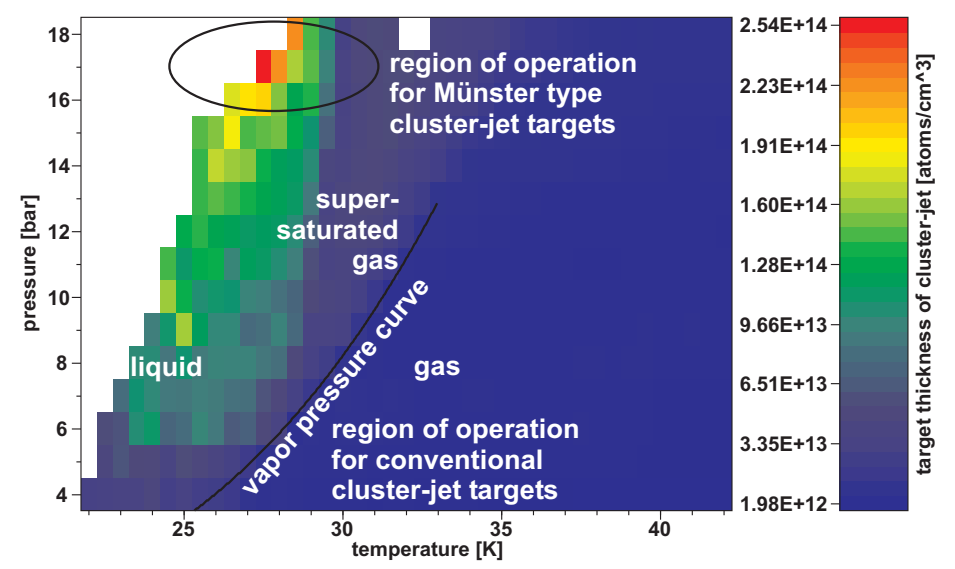

FIGURE 1. Target thickness of the target facility in Münster as function of the gas pressure and temperature. Shown are also the regions of operation for conventional targets compared to the Münster type.

\section{CLUSTER-JET TARGET FACILTY IN MÜNSTER}

Since the distance $d$ between between nozzle and interaction point is connected to the target thickness $n_{T}$ via the formula $n_{T} \propto d^{-2}$ it is remarkable that the target thickness of targets listed in the first three columns of table 1 are in the same range although the distance at ANKE and COSY-11 is enlarged by a factor of two. This shows the advantages of the Münster type targets which are operated in a regime where the hydrogen is supersaturated before the nozzle (see figure 1) leading to a drastic increase in target thickness.

Future experiments in hadron physics like the PANDA experiment at FAIR [3] will use $4 \pi$-detectors leading to a drastic increase in the distance between target and interaction point. In the case of the PANDA experiment the distance will be approximately two meters. In order to have the same target thickness as used in the established experiments it is necessary to increase the target thickness by at least one order of magnitude. In order to test the feasibility of such an increase in target thickness a complete target facility was build up in Münster which has a distance of $2.1 \mathrm{~m}$ between nozzle and interaction point. This distance represents the worst case scenario for the PANDA detector. Figure 2 shows a CAD drawing of the current setup in Münster. The cluster-jet produced in the cluster source traverses a vacuum chamber which is placed at the distance where the 


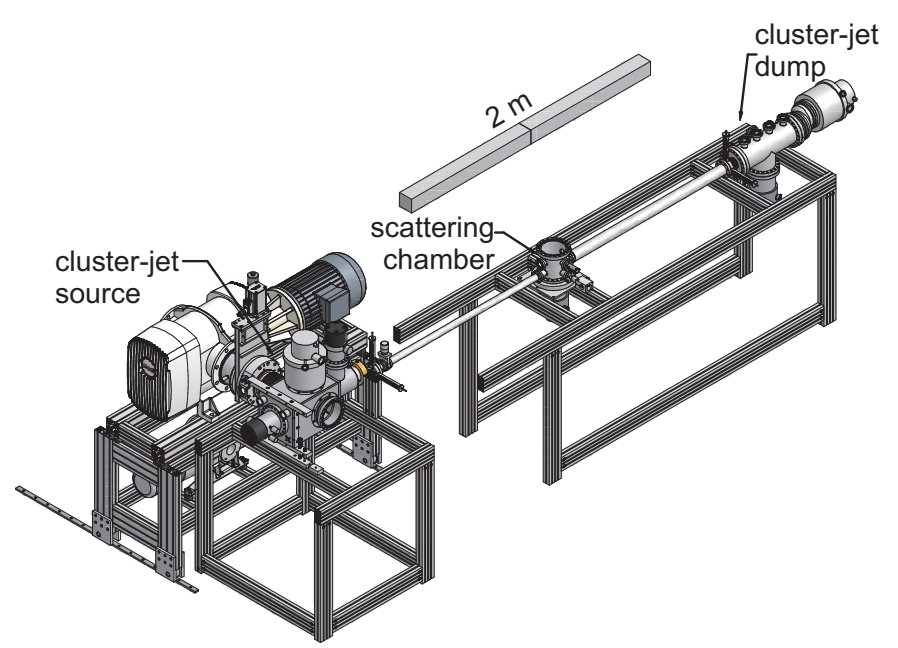

FIGURE 2. CAD drawing of the cluster-jet target used in Münster.

beam-target interaction would take place in the PANDA detector and is finally collected and pumped in the beam dump.

There are two parameters which can be changed when the cluster-jet target is in operation, namely the gas temperature and gas pressure before the nozzle. In figure 1 the target thickness measured in the scattering chamber is shown as function of these two parameters. The target thickness increases with increasing pressure and decreasing temperature. After detailed studies several improvements had been made in order to be able to operate at the point of highest thickness, e.g. the pumping speeds at the collimator and the skimmer chamber have been increased and the gas system has been modified to reduce heat losses.

The target thickness is measured by inserting a small rod into the cluster-jet beam stopping the clusters colliding with the rod which leads to a pressure increase in the scattering chamber. From the measured pressure increase the cluster-jet flow $\Phi$ can be calculated. Together with the cross section $A$ between rod and cluster-jet and the velocity $v_{j e t}$ the areal target thickness $n_{T}$ can be calculated

$$
n_{T}=\frac{\Phi}{A \cdot v_{j e t}} .
$$

Up to the end of last year the cluster velocity was calculated from the theoretically estimated maximum velocity of the gas leaving the laval nozzle. Measurements performed at the E835 experiment at FERMILAB confirmed this assumption [4] for the operation regime in the gaseous part above the vapor pressure curve. Since the Münster type target operates at much higher pressures and lower temperatures it had to be tested whether this assumptions holds also for the target setup used in Münster. Therefore, velocity measurements were performed using a time-of-flight technique where the clusters were ionized using a pulsed electron gun situated near the exit of the cluster source and detected with a channeltron at the end of the cluster-jet dump. First preliminary results of these measurements are show in figure 3 where the cluster velocity is plotted as function of the gas temperature at a constant gas pressure. In the region above the vapor pressure 


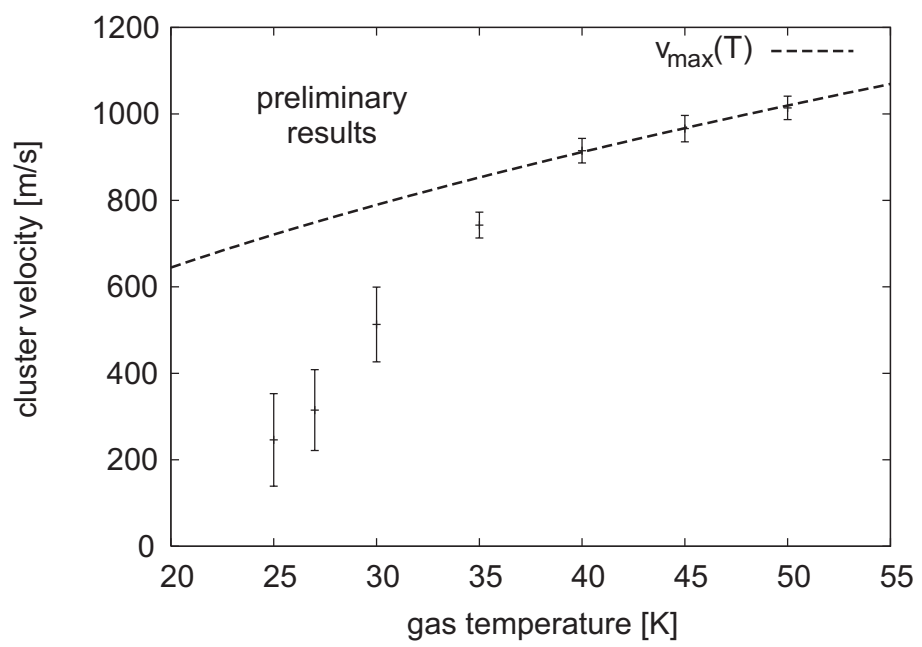

FIGURE 3. Velocity of the clusters measured using a TOF technique as function of the gas temperature at a constant gas pressure of 8 bar.

curve the measured velocity agrees well with the maximum gas velocity, but at lower temperatures the measured velocity is by a factor 2 to 3 lower than the prediction. This leads to an increase of the real target thickness by the same factor.

The maximum target thickness shown in figure 1 was calculated using the maximum gas velocity as cluster velocity. Considering the measured velocities the real target density at the interaction point turns out to be larger by a factor of 2-3. Due to this a target thickness of at least $4 \cdot 10^{14}$ atoms $/ \mathrm{cm}^{2}$ was achieved at a distance of $2.1 \mathrm{~m}$ between nozzle and interaction point. Further density increases are currently limited by the finite cooling power of the present setup.

\section{PROTOTYPE FOR PANDA}

After achieving the first goal of increasing the cluster-jet target thickness by a factor of 10 a new cluster source is currently build up which should be able to achieve lower temperatures and therefore higher target thicknesses. This new setup is at the same time a prototype for a cluster-jet target which can be installed at PANDA. The new setup will have a much more powerful coldhead for the gas cooling and will have improvements in the heat insulations. It will be possible to move the skimmer and the collimator in order to optimize the beam optics. Densities of $10^{15}$ atoms $/ \mathrm{cm}^{2}$ in combination with PANDA geometry are expected

\section{REFERENCES}

1. S. Brauksiepe, et al., Nucl. Instrum. Methods A 376, 397-410 (1996).

2. H. Dombrowski, et al., Nucl. Instrum. Methods A 386, 228-234 (1997).

3. PANDA Collaboration, Technical progress report, Tech. rep., FAIR (2005).

4. D. Allspach, et al., Nucl. Instrum. Methods A 410, 195-205 (1998). 